\title{
RESISTANCE MANAGEMENT IN THE 21st CENTURY: AN ENTOMOLOGIST'S POINT OF VIEW
}

\author{
M.A. CAPRIO ${ }^{1}$ and D.M. SUCKLING ${ }^{2}$ \\ ${ }^{1}$ Department of Entomology and Plant Pathology, Mississippi State University, \\ Mississippi State, MS 39792-9775 \\ ${ }^{2}$ HortResearch, PO Box 51, Lincoln, Christchurch, NZ
}

\begin{abstract}
It is probable that pest control will increasingly emphasize biotechnology as we approach the 21 st century. While biotechnology promises enormous benefits in terms of accurately placing the toxin in the field, even to the point of limiting expression of the toxin to specific plant structures at specific phenological stages, the very effectiveness of these strategies may also result in rapid adaptation by insect pests. The evolution of resistance can be delayed by tactics such as providing refuges, pyramiding toxins and combining transgenic crops with alternatives strategies, such as mating disruption. In most cases, these strategies have only been examined in models of single crop systems, but most transgenic crops will exist in a mosaic of crops that may contain either the same gene expressed at different levels or different genes with various levels of cross-resistance. Resilient resistance management strategies will need to consider insect movement and exposure to these toxins within this mosaic. Recent models of single crop systems suggest that toxin mixtures in systems with external ("out-of-field") refuges, combined with the use of economic thresholds, can significantly delay resistance. These simulations suggest that designing external refuges to manipulate source-sink dynamics can be more important in delaying resistance than the generation of nonrandom mating by the partial isolation of the refuges. While crossresistance between toxins in these single crop simulations did speed resistance evolution, the ranking of the different strategies did not differ over large ranges of cross-resistance (e.g. cross-resistance had a negative impact on all strategies). As these simulation models begin to examine mosaics of multiple crops, they must contend with the impact of crossresistance among multiple toxins in different crops, multiple resistance management strategies for the same toxins in different crops and the changes in demographics that may alter the relative importance of specific habitats over time.
\end{abstract}

Keywords: resistance, transgenic, genes, strategy, pest management

\section{RESISTANCE MANAGEMENT}

As the technology to introduce useful foreign genes into plants using recombinant technology improves, we will be faced with difficult questions regarding the design and deployment of transgenic crops. Seed producers will be limited in the number of varieties they can maintain, so decisions must be made on what combinations of genes will be incorporated into commercial varieties (I will refer to different combinations of transgenes backcrossed into the same non-transgenic variety as different varieties for convenience sake). These decisions will depend on compatibilities between genes, grower requirements (which may vary with geographic region), and resistance management strategies. In the latter case, the resistance management strategies are likely to vary with the targeted pest (insect, weed, or pathogen) because of the unique biological characteristics of each pest. A refuge strategy may often be a useful strategy to delay resistance evolution in insects to transgene produced toxins, but is unlikely to delay resistance to a gene that provides 
protection against a plant pathogen, as a prerequisite for successful use of the refuge strategy is sexual reproduction, which occurs only rarely in most plant pathogens. In such cases, decisions on which varieties to produce should incorporate information about the compatibility of resistance management strategies.

Resistance management options for transgenic crops will depend upon whether a single gene is being utilized among multiple crops, multiple genes are being used in a single crop, or multiple genes are being used among multiple crops (Table 1).

\section{TABLE 1: Potential problem areas that arise when utilizing transgenic plants for resistance to pests.}

$\begin{array}{cc}\text { A } & \text { Single gene shared among multiple crops } \\ \text { B } & \text { Multiple genes used within a single crops } \\ 1 \text { - Genes target the same pest } & 2 \text { - Genes target multiple pests } \\ & \\ \text { C } & \text { Multiple genes shared among multiple crops } \\ 1 \text { - Cross-resistance between genes } & 2 \text { - Cross-resistance not present }\end{array}$

\section{A) Single Gene}

While it is difficult to anticipate all potential problems with the implementation of transgenic crops, it is probable that some of the most vexing problems occur with the utilization of a single gene in multiple crops. If no major pests move among those crops, resistance management is relatively straightforward, though certainly not simple. The resistance management strategies that are effective for single toxins (reviewed by Tabashnik 1990) should be effective in these cases. In contrast, when pests move among different crops that incorporate the same gene, developing effective resistance management strategies may be quite difficult. Caprio (1994) used simulations to examine trade-offs that might occur in an agroecosystem in which the same toxin gene was utilized differently by two groups of growers. One group of growers used foliar applications of formulated $\mathrm{Bt}$ that included toxins produced by the same gene used in transgenic plants (and that produced the toxin endogenously) by a second group of growers. Each group of growers could either choose to cooperate or not cooperate with a resistance management strategy. Transgenic growers could cooperate with resistance management by planting a refuge, while foliar producers could cooperate by reducing their Bt spray usage per year $66 \%$ (from six sprays to two, with an alternative toxin used for the remaining four sprays). The strategy use by foliar producers works best if there are fitness costs associated with resistance. In addition, Caprio (1997) has shown that such a strategy allows more time for migration to occur between refuges and transgenic fields, contributing to a delay in resistance evolution if refuges are present. While it is difficult to determine when a transgenic producer will consider resistance management a success in this system (although see Roush and Powles 1996), for the foliar producer, success is easier to quantify. Resistance must take at least three fold longer to evolve when utilizing the resistance management strategy. If resistance evolves in less time, these growers would not have ben able to use the same number of applications of this spray before resistance evolved as they would have had, had they not cooperated with resistance management. A small subset of the data represents some of the problems (Table 2). In no case were the conditions for the successful cooperation of the foliar producers ever satisfied. While the strategy the foliar producers employed may have accrued some susceptibility in the population, this shared resource was rapidly depleted by transgenic producers. As might be expected, when fitness costs were increased in the simulations, it became more likely that conditions for cooperation by the foliar producers could be satisfied. This simple two strategy agroecosystem demonstrates that in more complex systems it is possible to design resistance management strategies that are not advantageous to all producers. 
Because growers utilized a shared resource (susceptibility to the same toxin), growers implementing a resistance management strategy may not receive as much benefit as they would expect in isolation. The inevitable result is that the shared resources may be unequally distributed among grower groups. This suggests that as a toxin is used among more crops, it will be more difficult to implement resistance management strategies that are fair to all producers.

\section{TABLE 2: A payoff matrix for resistance management cooperation decisions for two groups of growers utilizing the same gene in two different fashions (foliar applications and transgenic plants). Data are the time (days) required for $50 \%$ of transgenic fields to have a resistance allele frequency greater than $50 \%$.}

\begin{tabular}{lcc}
\hline Transgenic producers show & \multicolumn{2}{c}{ Foliar producers } \\
& Cooperation & Non-cooperation \\
\hline Cooperation & 1961.0 & 1187.7 \\
Non-cooperation & 1434.4 & 651.0 \\
\hline
\end{tabular}

\section{B) Multiple genes within a single crop}

When multiple toxins are used in plants, they may be used to target the same pest or they may be targeted at different organisms. When the toxins are targeted at the same pest, the effectiveness of a multiple-toxin strategy will depend upon the presence of refuges. Caprio (1997) examined the evolution of resistance in Heliothis virescens to cotton treated with foliar applications of Bt using a number of alternative resistance management strategies in the presence of both internal (within field) and external (outof-field) refuges. In the absence of refuges, using full rates of 2 toxins can lead to resistance to both toxins in approximately the same amount of time it would have taken for resistance to evolve to 1 toxin (Fig. 1) (Caprio 1997; see also Gould 1986). In the presence of refuges, however, full-rate mixtures were very effective as a resistance management strategy (Fig. 2). Though these simulations also incorporated economic thresholds, they clearly show that the effect of mixtures is strongly conditioned by the presence of refuges. In the presence of a 5 percent refuge, full-rate mixtures delayed resistance development by almost 10-fold compared to a single-toxin strategy, despite that fact that the dose was relatively low and only killed on average about $20 \%$ of susceptible individuals. Thus, use of multiple toxins in transgenic plants will probably only be an effective strategy if reliable, nontoxic refuges are available, either in the form of alternate, perhaps wild, hosts, or through the deliberate planting of plants that do not express the toxin.

When the multiple toxins in the plant target different pests, the primary concern becomes the proper concurrent implementation of resistance management strategies for both toxins. In addition to marketing strategies, decisions regarding the appropriate transgenic varieties to produce commercially should incorporate the needs of resistance management. In general, resistance management strategies for insects, weeds and plant pathogens are quite different and an acceptable strategy for one pest may not be suitable for another. As mentioned previously, a refuge strategy appears to be quite promising for resistance management of toxins targeted at insects, but is probably unsatisfactory for genes targeted at plant pathogens, at least those that do not reproduce sexually. Thus, plants that do not contain insecticidal genes may be important for the deployment of refuges, but it is likely that the plants in the refuge should contain the genes that target plant pathogens such as viruses. The refuges would otherwise harbor large amounts of genetic variation that might speed up the evolution of resistance by the pathogens (Uyenoyama 1986).

\section{C) Multiple genes among multiple crops}

The final combination occurs when multiple genes are used among multiple crops. It is important to remember that problems only arise in this case when pests move among 


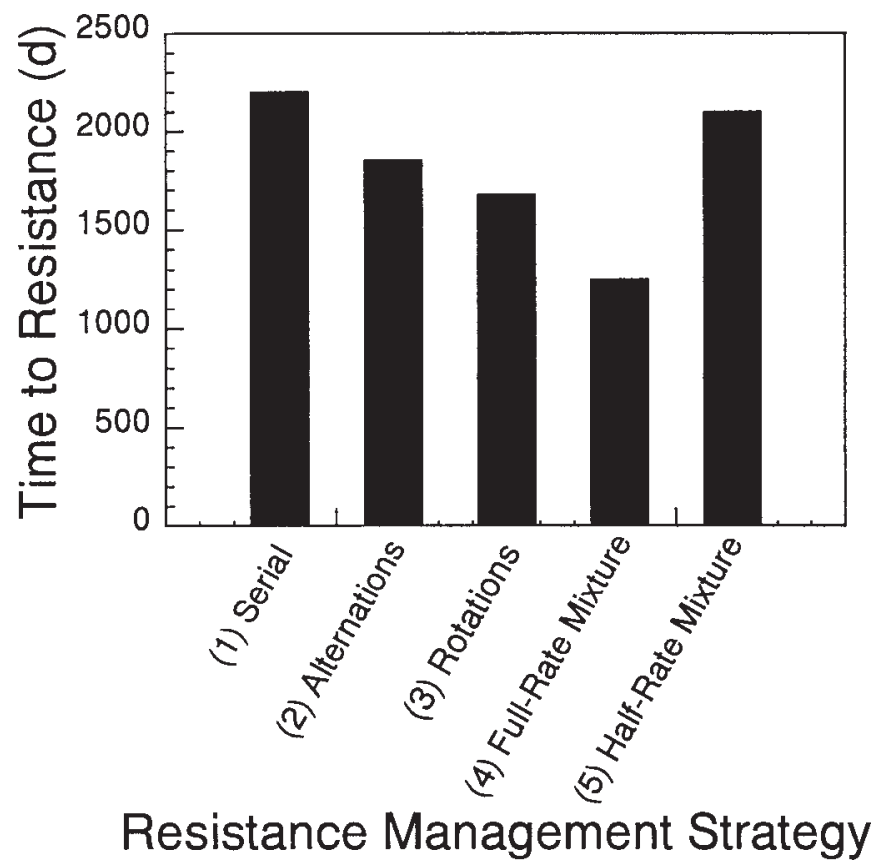

FIGURE 1: Time taken (days) for $50 \%$ of transgenic fields to have a resistance allele frequency greater than $50 \%$ when fields were treated every 30 days with a range of resistance management tactics using multiple toxins. The strategies use were: (1) Serial: use one toxin until resistance develops, then use the second toxin; (2) Alternation: Use two toxins in alternation, all fields use the same toxin; (3) Mosaic: use two toxins in alternation, but $50 \%$ of fields use each toxin each year; (4) Fullrate mixture: use two toxins simultaneously, each at the same fullrate used in the serial strategy simulations; (5) Half-rate mixture: use two toxins simultaneously at rates so that mortality from the mixture is the same as the mortality from a single toxin used at a full-rate. The generation time for the simulated pest (H. virescens) was 28.1 days.

the crops. If crops are ecologically isolated from each other, there are likely to be few complications beyond those of resistance management to single toxins. When insects move among crops, the problems that arise depend upon the presence of cross-resistance.

It may also be necessary to identify the usefulness of a particular gene in various crops, as it is quite conceivable that genes may vary in utility in different crops. It is possible that a gene that is of critical importance in one crop can be inserted into a second crop as a low value add-on. The use of the gene in the second crop could well hasten resistance evolution in the primary crop. This is particularly difficult because it means that there may be no purely objective way to determine the best way to utilize transgenes, as the optimal solution may depend on value judgements. In the mid-South of the USA, plantings of corn modified to produce Bacillus thuringiensis (Bt) toxins are presently limited by the Environmental Protection Agency (E.P.A) because of a concern of crossresistance between the toxins utilized in Bt-transgenic cotton and corn. In this case, the E.P.A. has determined for the present that the utility of the Bt genes in cotton (and the 


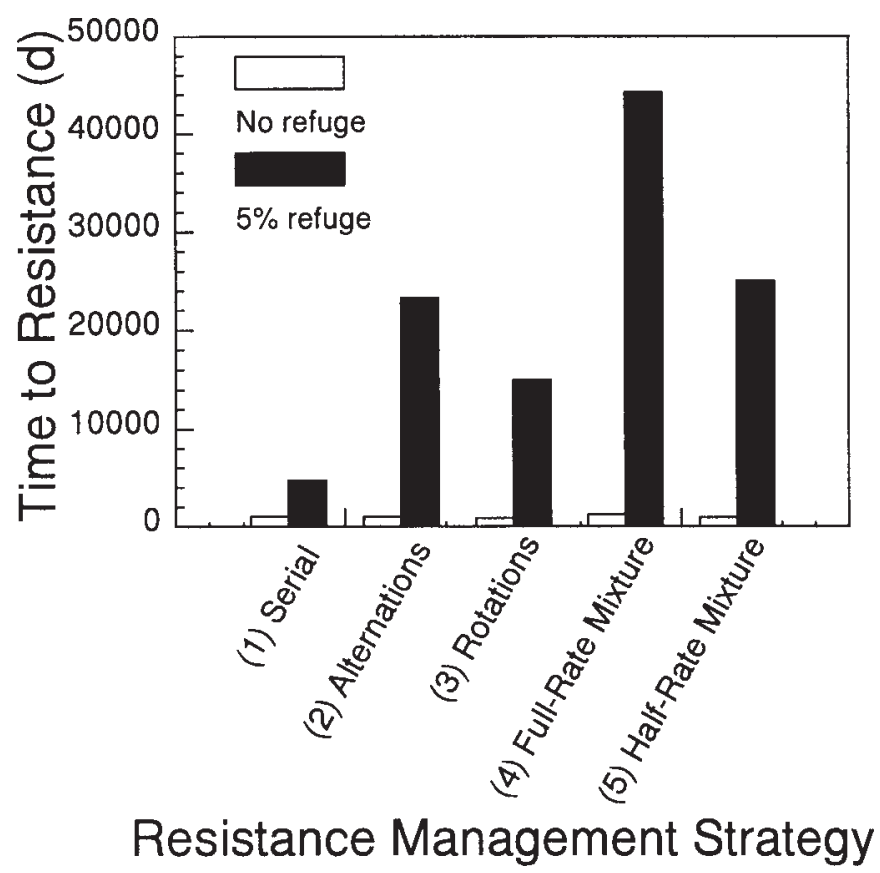

FIGURE 2: The time taken (days) for $50 \%$ of the treated fields to experience control failures when foliar applications of a pesticide were applied when an economic threshold was exceeded. A control failure occurred when the population size exceeded the economic threshold by ten fold. Resistance management strategies as in Fig. 1.

increase in environmental quality due to reduced pesticide use), outweighs the increase in yields that might be expected from implementing Bt-corn (at present, few spray applications are directed at the targets of the Bt toxins in corn). In the northern USA, where Bt-cotton is not present, no such restrictions on Bt-corn have been established.

In the absence of cross-resistance, each crop should provide a suitable independent resistance management strategy. It seems likely that in the absence of interactions between the ecosystems, treating each ecosystem independently is the best strategy. Care should be taken to ensure that the resistance management strategies are independent. For example, wild hosts might be a sufficient refuge for a single crop, but insufficient for multiple crops. In such cases, it will be difficult to allocate such shared resources. In other cases, an apparent refuge may not function as such if no movement or mating occurs between individuals in the different habits. For example, fall armyworm (Spodoptera frugiperda) is a pest on sweet corn in Southern Florida. Large acreages of Bermuda grass are also present in this area, and it has been suggested that the considerable numbers of fall armyworm that develop on that host should provide a refuge for transgenic sweet corn. However, Pashley $(1986 ; 1987)$ has suggested that the strain of fall armyworm on Bermuda grass (and rice) is reproductively isolated from the strain that predominates in corn and may even be a sibling species. In this case, reliance on populations of fall armyworm from Bermuda grass to provide a refuge for transgenic corn could lead to rapid evolution of resistance in this serious pest.

When cross-resistance between the toxins used in the multiple crops occurs, susceptibility again becomes a shared resource and problems similar to using a single 
gene among multiple crops develops. We have been developing simulations of such a situation for the apple/clover ecosystem in New Zealand. In particular, we are examining the potential for resistance evolution in the lightbrown apple moth (Epiphyas postvittana) to apple trees and clover that have been modified to express related Bt endotoxins. We assume that cross-resistance is not complete, such that a locus that provides resistance to the toxin expressed in apple trees only provides partial resistance to the toxin expressed in clover. Similarly, the genetic locus that provides resistance to the toxin in clover only provides partial resistance to the toxin in the apple trees. This might best be termed reciprocal partial cross-resistance. Wearing and Hokkanen (1984) suggested that crossresistance between Cry genes used in clover and apples could have implications for horticultural crops. These simulations support the position that high levels of crossresistance can increase the rate at which resistance will develop (Fig. 3). Interestingly, low levels of cross-resistance actually delay resistance because in this case, two loci respond to selection and the response at each locus is somewhat slower. The single most important factor in altering the rate of resistance to the toxin in the transgenic apple orchards was not, however, cross-resistance but rather the choice to produce toxins in the clover. Even in the absence of cross-resistance, the deployment of transgenic clover greatly reduced the effective size of the refuge for the light brown apple moth and dramatically increased the rate at which resistance evolved, at least in our simulations. The non-transgenic clover was quite effective as a refuge despite that fact that the carrying capacity in the apple orchards was 17 -fold greater than the carrying capacity in the clover pastures and under normal conditions the populations in the clover would be relatively small. Though we simulated a second refuge in the understory of the transgenic orchards, this refuge was relatively ineffective because individuals in the orchard

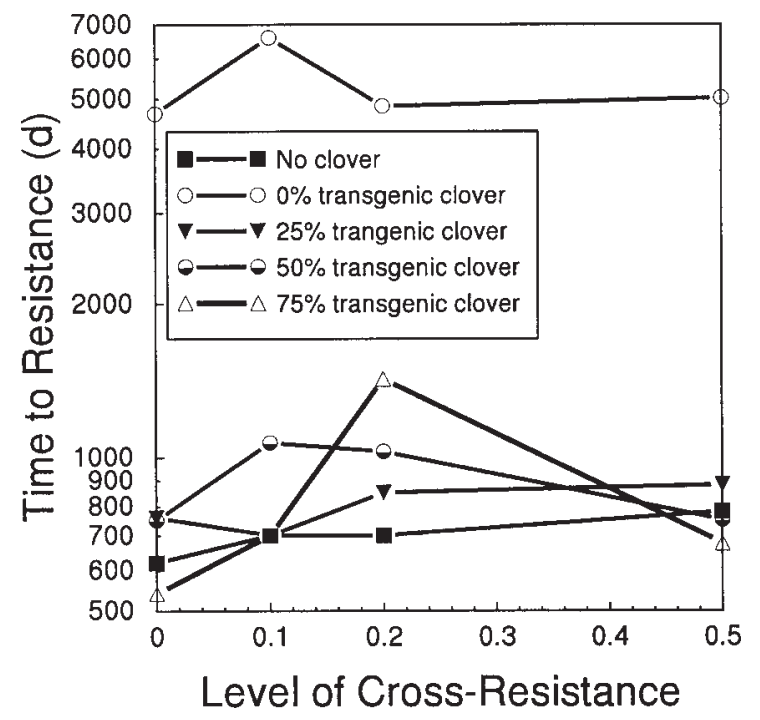

FIGURE 3: The impact of symmetric, partial cross-resistance on resistance evolution in the light brown apple moth (Epiphyas postvittana) in a multi-crop simulation of transgenic cultivars. The time to resistance is the number of days before $50 \%$ of the treated fields had resistance allele frequencies greater than $50 \%$. The carrying capacity for LBAM larvae in clover pastures was 3000 , and in orchards it was 50,000 . 
oviposited most eggs on transgenic trees where they were unlikely to survive. This is consistent with previous results for internal and external refuges to transgenic apple orchards (Caprio and Suckling 1995). The population in the understory refuge had a much lower growth rate because the transgenic trees were acting as a large population sink. In contrast, the populations in the non-transgenic clover pastures had higher growth rates (there was no in-field sink), and were therefore more effective over the long term than the understory refuges, despite the fact that the clover pasture habitats had low carrying capacities and were less common than the apple orchards. These data are consistent with previous work (Caprio unpubl. data) that refuges for transgenic crops must be large enough the ensure that populations in the refuges have a positive growth rate.

These data also suggest that the genetics of cross-resistance may be less important than the population dynamics issues that arise when genes are put in multiple crops. If the genes are seen to have equal utility in both crops (leaving the gene out of one crop is not an option), then cross-resistance can be a major problem. In those situations however, where the gene is of less utility in one or more of the crops, serious consideration should be given to the utility of those crops as refuges to crops where the transgenes are felt to be critical. Such choices are based on value judgments, and values will vary with grower groups, seed producers, and consumers. Some method to resolve conflicts among these different groups will have to be developed if we hope to rationally utilize the potential of transgenic crops.

\section{REFERENCES}

Caprio, M.A., 1994.Bacillus thuringiensis gene deployment and resistance management in single- and multi-tactic environments. Biocont. Sci. Tech. 4: 487-497.

Caprio, M.A., 1997. Evaluating resistance management strategies for multiple-toxins in the presence of external refuges.J. Econ. Entomol: (submitted).

Caprio, M.A. and Suckling, D.M., 1995. Mating disruption reduces the risk of resistance development to transgenic apple orchards: simulations of the light brown apple moth. Proc. 48th N.Z. Plant Prot. Conf. : 52-58.

Gould, F., 1986. Simulation models for predicting durability of insect-resistant germ plasm: a deterministic diploid, two locus model. Environ. Entomol 15: 1-10.

Pashley, D.P., 1986. Host-associated genetic differentiation in fall armyworm (Lepidoptera: Noctuidae): a sibling species complex? Ann. Entomol. Soc. Am. 79: 898-904.

Pashley, D.P. and Martin, J.A. 1987. Reproductive incompatibility between host strains of fall armyworm (Lepidoptera: Noctuidae). Ann. Entomol. Soc. Amer. 80: 731733.

Roush, R.T. and Powles, S.B., 1996. Pesticide resistance: why be concerned? Pp. 3-28. In: Pesticide Resistance: Prevention and Management. G.W. Bourdot and D.M. Suckling (Eds.). NZ Plant Protection Society, Rotorua, NZ.

Tabashnik, B.E., 1990. Modeling and evaluation of resistance management tactics. Pp. 153-182. In: Pesticide Resistance in Arthropods. R.T. Roush and B.E. Tabashnik (Eds.). Chapman and Hall, New York.

Uyenoyama, M.K., 1986. Pleiotropy and the evolution of genetic systems conferring resistance. Pp. 207-221. In: Pesticide Resistance: Strategies and Tactics For Management. National Res. Council (Ed.). National Academy Press, Washington, D.C.

Wearing, C.H. and Hokkanen, H.M.T., 1984. Pest resistance toBacillus thuringiensis: Case studies of ecological crop assessment for Bt gene incorporation and strategies of management. Biocont. Sci. Tech. 4: 573-590. 